Supporting Information for

\title{
Periodic polarization waves in a strained, highly polar ultrathin $\mathrm{SrTiO}_{3}$
}

Yunlong Tang ${ }^{1}$, Yinlian $\mathrm{Zhu}^{1,2}$, Bo $\mathrm{Wu}^{2}$, Yujia Wang ${ }^{1}$, Lixin Yang ${ }^{1}$, Yanpeng Feng ${ }^{2,3}$, Minjie $\mathrm{Zou}^{2,3}$, Wanrong Geng ${ }^{2,3}$, and Xiuliang $\mathrm{Ma}^{1,4 *}$

${ }^{1}$ Shenyang National Laboratory for Materials Science, Institute of Metal Research, Chinese Academy of Sciences, Wenhua Road 72, 110016 Shenyang, China

${ }^{2}$ Songshan Lake Materials Laboratory, Dongguan, Guangdong 523808, China

${ }^{3}$ Institute of Physics, Chinese Academy of Sciences, Beijing 100190, China

${ }^{4}$ State Key Lab of Advanced Processing and Recycling on Non-ferrous Metals, Lanzhou University of Technology, Langongping Road 287, 730050 Lanzhou, China

*Correspondence should be addressed to X. L. Ma (xlma@imr.ac.cn) 


\section{Materials and Methods}

\section{Film deposition details}

The thin films were deposited by pulsed laser deposition (PLD), using a Lambda Physik LPX 305i KrF $(\lambda=248 \mathrm{~nm})$ excimer laser. The $\mathrm{PbTiO}_{3}$ targets were $3 \mathrm{~mol} \% \mathrm{~Pb}$-enriched sintered ceramics. The target-substrate distance was $40 \mathrm{~mm}$. The background pressure was $10^{-5} \mathrm{~Pa}$. During the growth of $\mathrm{PbTiO}_{3}$, the substrate temperature was kept at $650^{\circ} \mathrm{C}$, with a laser energy density of $2 \mathrm{Jcm}^{-2}$, a laser repetition rate of $5 \mathrm{~Hz}$ and under an oxygen pressure of $20 \mathrm{~Pa}$. For the growth of $\mathrm{SrTiO}_{3}$ layers, the substrate temperature was also $650^{\circ} \mathrm{C}$, with a laser energy density of $1 \mathrm{Jcm}^{-2}$, a laser repetition rate of $2 \mathrm{~Hz}$ and under an oxygen pressure of $8 \mathrm{~Pa}$. Before deposition, the substrate was pre-heated at $750^{\circ} \mathrm{C}$ for 5 min to clean the substrate surface and then cooled down to the growth temperature $\left(10^{\circ} \mathrm{C} / \mathrm{min}\right)$. The laser was focused on the ceramic target for 30 min pre-sputtering to clean the target surface. After deposition, the film was annealed at $650^{\circ} \mathrm{C}$ in an oxygen pressure of $5 \times 10^{4} \mathrm{~Pa}$ for $10 \mathrm{~min}$, and then cooled down to room temperature at a cooling rate of about $5^{\circ} \mathrm{C} / \mathrm{min}$.

\section{Strain analysis by Geometric Phase Analysis (GPA)}

Large scale strain fields were deduced from the HAADF-STEM lattice images using custom plugins of GPA ${ }^{12,19,20}$ for Gatan DigitalMicrograph. The GPA is an effective approach to determine crystal lattice variations in a large area of an image. The visualization of the strains and lattice rotations was carried out using the Gatan DigitalMicrograph software.

\section{HAADF-STEM (TEM) experiment and the determination of cation off-center displacements}

The samples for the STEM and TEM experiments were prepared by slicing, gluing, grinding, dimpling, and finally ion milling. A Gatan PIPS was used for the final ion milling. Before ion milling, the samples were dimpled down to less than $20 \mu \mathrm{m}$. The final ion milling voltage was less than $1 \mathrm{kV}$ to reduce ion beam damage. HAADF-STEM images were recorded using aberration-corrected scanning transmission electron microscopes (Titan Cubed 60-300kV microscope (FEI) fitted with a high-brightness field-emission gun (X-FEG) and 
double Cs corrector from CEOS, and a monochromator operating at $300 \mathrm{kV}$ ). The beam convergence angle is $\sim 25 \mathrm{mrad}$, yielding a probe size of less than $0.10 \mathrm{~nm}$. Low-magnification diffraction contrast TEM images of ferroelectric domain patterns were recorded using a common transmission electron microscope (Tecnai G2 F30 microscope (FEI) operating at $300 \mathrm{kV}$ ).

For determining the $\mathrm{Ti}^{4+}$ displacement vectors, noise in the obtained HAADF-STEM images was reduced by Fast Fourier Transform (FFT) filtering, using a lattice mask and a low-pass annular mask restricted to the instrument resolution limit of the image $\mathrm{e}^{12,19,30,31}$. The atom positions were determined accurately by fitting them as 2D Gaussian peaks by using Matlab, thus the lattice spacing and $\mathrm{Ti}^{4+}$ shifts $\left(\delta_{T i}\right)$ were deduced. The $\delta_{T i}$ were calculated as a vector between each $\mathrm{Ti}^{4+}$ and the center of mass of its four nearest $\mathrm{A}$-site neighbor $\mathrm{Pb}^{2+}$ or $\mathrm{Sr}^{2+}$. As the $\mathrm{PbTiO}_{3}$ crystal undergoes a paraelectric to ferroelectric phase transition, the oxygen octahedron is shifted along the $c$ axis of the $\mathrm{PbTiO}_{3}$ unit-cell (Figure S1). The visualization of the $2 \mathrm{D} \delta_{T i}$ vectors was carried out using Matlab.
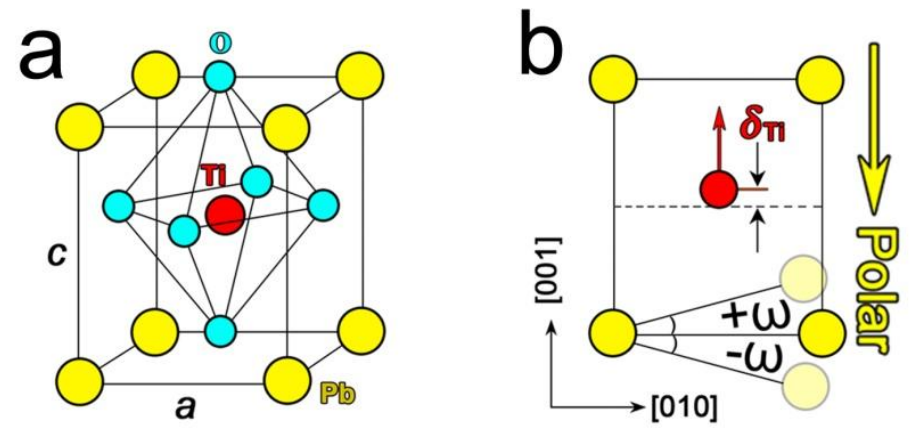

Figure S1. The atomic scale structure of the $\mathrm{PbTiO}_{3}$ unit-cell. (a) Schematic of the unit-cell of ferroelectric $\mathrm{PbTiO}_{3}$. (b) Projection of the $\mathrm{PbTiO}_{3}$ unit-cell along [100] direction. Yellow, red and light blue circles represent $\mathrm{Pb}, \mathrm{Ti}$ and $\mathrm{O}$ atoms, respectively.

\section{Phase field Modeling}

The polarization distribution in PTO/STO/PTO multilayer films was studied by phase field simulations. The order parameters are chosen as the three components of spontaneous polarizations. The system's free energy is composed of bulk, gradient, elastic and electrostatic ones:

$$
F=\int_{V}\left[f_{\text {bulk }}\left(P_{i}\right)+f_{\text {grad }}\left(P_{i, j}\right)+f_{\text {elas }}\left(P_{i}, \varepsilon_{k l}\right)+f_{\text {elec }}\left(P_{i}, E_{i}\right)\right] d V
$$


The first term is the bulk energy density:

$$
\begin{aligned}
& f_{\text {bulk }}=\alpha_{1}\left(P_{1}^{2}+P_{2}^{2}+P_{3}^{2}\right)+\alpha_{11}\left(P_{1}^{4}+P_{2}^{4}+P_{3}^{4}\right) \\
& +\alpha_{12}\left(P_{1}^{2} P_{2}^{2}+P_{2}^{2} P_{3}^{2}+P_{1}^{2} P_{3}^{2}\right)+\alpha_{111}\left(P_{1}^{6}+P_{2}^{6}+P_{3}^{6}\right) \\
& +\alpha_{112}\left[P_{1}^{4}\left(P_{2}^{2}+P_{3}^{2}\right)+P_{2}^{4}\left(P_{1}^{2}+P_{3}^{2}\right)+P_{3}^{4}\left(P_{1}^{2}+P_{2}^{2}\right)\right]+\alpha_{123} P_{1}^{2} P_{2}^{2} P_{3}^{2}
\end{aligned}
$$

The second term is the gradient energy density:

$$
\begin{aligned}
& f_{\text {grad }}=\frac{1}{2} G_{11}\left(P_{1,1}^{2}+P_{2,2}^{2}+P_{3,3}^{2}\right)+G_{12}\left(P_{1,1} P_{2,2}+P_{2,2} P_{3,3}+P_{1,1} P_{3,3}\right) \\
& +\frac{1}{2} G_{44}\left[\left(P_{1,2}+P_{2,1}\right)^{2}+\left(P_{2,3}+P_{3,2}\right)^{2}+\left(P_{3,1}+P_{1,3}\right)^{2}\right]
\end{aligned}
$$

The third term is the elastic energy density:

$$
f_{\text {elas }}=\frac{1}{2} C_{i j k l}\left(\varepsilon_{i j}-\varepsilon_{i j}^{0}\right)\left(\varepsilon_{k l}-\varepsilon_{k l}^{0}\right)
$$

where $\varepsilon_{i j}$ is the total strain and $\varepsilon_{i j}^{0}$ is the spontaneous strain. Their difference is the elastic strain. The spontaneous strain is related to the polarization by the electrostrictive coefficients:

$$
\begin{aligned}
& \varepsilon_{11}^{0}=Q_{11} P_{1}^{2}+Q_{12}\left(P_{2}^{2}+P_{3}^{2}\right) \\
& \varepsilon_{22}^{0}=Q_{11} P_{2}^{2}+Q_{12}\left(P_{1}^{2}+P_{3}^{2}\right) \\
& \varepsilon_{33}^{0}=Q_{11} P_{3}^{2}+Q_{12}\left(P_{2}^{2}+P_{1}^{2}\right) \\
& \varepsilon_{23}^{0}=Q_{44} P_{2} P_{3} \\
& \varepsilon_{13}^{0}=Q_{44} P_{1} P_{3} \\
& \varepsilon_{12}^{0}=Q_{44} P_{1} P_{2}
\end{aligned}
$$

The last term is the electrostatic energy density:

$$
f_{\text {elec }}=-\frac{1}{2} \varepsilon_{0} \varepsilon_{b} E_{i} E_{i}-E_{i} P_{i}
$$

where $\varepsilon_{0}$ is the permittivity of vacuum and $\varepsilon_{b}$ is the background relative dielectric constant.

A mesh of $128 \times 2 \times N_{z}$ is adopted to build the PTO/STO/PTO sandwich structure. The grid spacings are $x_{0}=y_{0}=1 \mathrm{~nm}, z_{0}=0.4 \mathrm{~nm}$. The thicknesses of the two PTO layers are both 25 grids $(10 \mathrm{~nm})$ and the middle STO layer varies from 2 grids $(0.8 \mathrm{~nm})$ to 30 grids $(12 \mathrm{~nm})$. The material parameters of PTO and STO are adopted from previous literatures ${ }^{14,44}$. At each PTO/STO interface, a layer of one grid thick $(0.4 \mathrm{~nm})$ is set as the transition layer whose 
material parameters are the averaged values of PTO and STO. Under the PTO/STO/PTO sandwich, a layer of substrate whose thickness is 20 grids $(8 \mathrm{~nm})$ is considered to simulate the elastic deformation of the substrate due to the presence of the grown film. The initial structure is the $a / c$ domains with the uniform polarization in both PTO and STO. The mechanical boundary condition is a mixed one: the top surface of the film is in a traction-free state, while the bottom of the deformable substrate is fixed. The short-circuit electric boundary condition is considered in the simulation.

We assume that the equilibrium of mechanical stress and electrical field is much faster than the evolution of domain structures. Thus, for each polarization configuration, the mechanical and electrical equilibrium equations

$$
\begin{aligned}
& \sigma_{i j, j}=0 \\
& D_{i, i}=0
\end{aligned}
$$

are solved to obtain the corresponding driving forces.

The evolution of polarizations is governed by the time-dependent Ginzburg-Landau equation:

$$
\frac{d P_{i}}{d t}=-L \frac{\delta F}{\delta P_{i}}
$$

We use the backward Euler method to trace the evolution of polarizations.

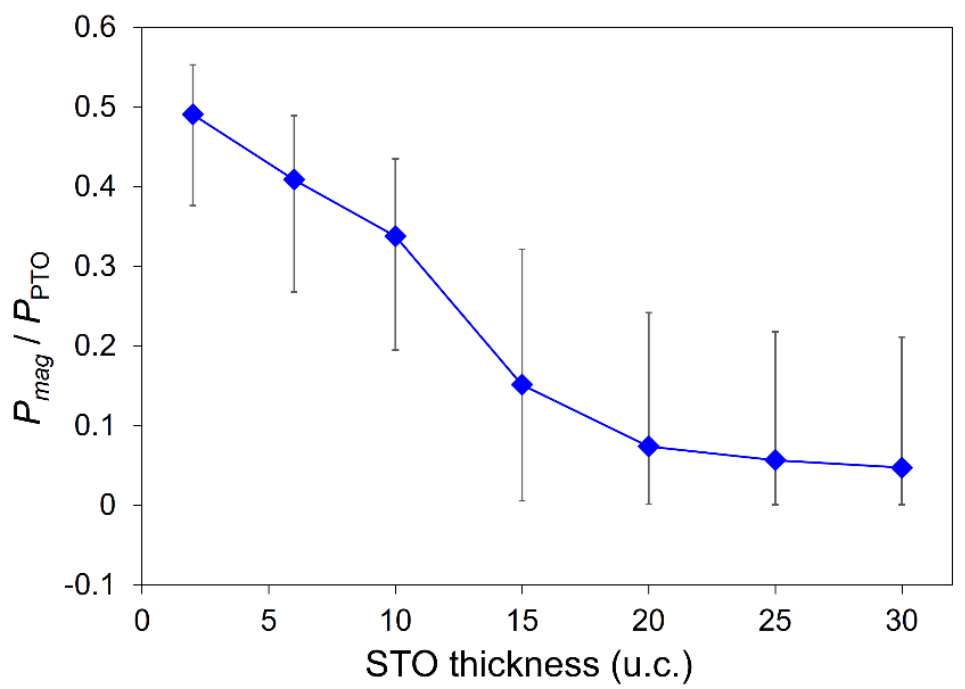

Figure S2. The evolution of averaged polarization magnitudes in the STO layers with the STO thicknesses obtained from the phase-field simulations. 
By using the above obtained phase-field simulations, the evolution of averaged polarization magnitudes in the STO layers with the thicknesses of the STO layers were deduced, as shown in Figure S2. The thicknesses of the STO layers change from 2 unit cells to 30 unit cells here. It was found that the obtained polarizations in the ultrathin STO layers degraded quickly as the STO layer gets thicker. Specially, when the STO layer is larger than 15 unit cells $(\sim 6 \mathrm{~nm})$, the averaged polarization magnitude in the STO layer will evolve into an insignificant value when compared with bulk PTO, which signifies that the critical thickness for the STO layer to be polar is about $6 \mathrm{~nm}$.

\section{Reference}

44. Li, Y. L. et al. Prediction of ferroelectricity in $\mathrm{BaTiO}_{3} / \mathrm{SrTiO}_{3}$ superlattices with domains. Appl. Phys. Lett. 91, 112914 (2007). 